\title{
2D Atomic Mapping of Oxidation States in Transition Metal Oxides by Scanning Transmission Electron Microscopy and Electron Energy-Loss Spectroscopy
}

\author{
Haiyan Tan, ${ }^{1, *}$ Stuart Turner, ${ }^{1}$ Emrah Yücelen, ${ }^{2,3}$ Jo Verbeeck, ${ }^{1}$ and Gustaaf Van Tendeloo ${ }^{1}$ \\ ${ }^{1}$ EMAT, University of Antwerp, Groenenborgerlaan 171, B-2020 Antwerpen, Belgium \\ ${ }^{2}$ FEI Company, Europe NanoPort, Achtseweg Noord 5, 5651 GG Eindhoven, The Netherlands \\ ${ }^{3}$ National Centre for HREM, Delft University of Technology, Lorentzweg 1, 2628 CJ Delft, The Netherlands \\ (Received 15 February 2011; revised manuscript received 27 July 2011; published 1 September 2011)
}

\begin{abstract}
Using a combination of high-angle annular dark-field scanning transmission electron microscopy and atomically resolved electron energy-loss spectroscopy in an aberration-corrected transmission electron microscope we demonstrate the possibility of $2 \mathrm{D}$ atom by atom valence mapping in the mixed valence compound $\mathrm{Mn}_{3} \mathrm{O}_{4}$. The $\mathrm{Mn} L_{2,3}$ energy-loss near-edge structures from $\mathrm{Mn}^{2+}$ and $\mathrm{Mn}^{3+}$ cation sites are similar to those of $\mathrm{MnO}$ and $\mathrm{Mn}_{2} \mathrm{O}_{3}$ references. Comparison with simulations shows that even though a local interpretation is valid here, intermixing of the inelastic signal plays a significant role. This type of experiment should be applicable to challenging topics in materials science, such as the investigation of charge ordering or single atom column oxidation states in, e.g., dislocations.
\end{abstract}

DOI: 10.1103/PhysRevLett.107.107602

PACS numbers: 79.20.Uv, 68.37.Ma, 75.25.Dk

In transition metal oxides, the oxidation state of the transition metal cations is of fundamental importance as the physical properties of many oxides are determined by the occupancy of the cation $d$ bands. Multiferroicity, for example, can be driven by charge ordering of these cations, as in $\mathrm{Fe}_{3} \mathrm{O}_{4}$ and $\mathrm{Pr}_{1-x} \mathrm{Ca}_{x} \mathrm{MnO}_{3}$ [1-3]. However, the exact mechanism of multiferroic behavior is not fully understood to date. A method allowing for direct mapping of cation valence states in these materials at atomic resolution should therefore provide further insight into the origin of multiferroicity.

Recently, atomic resolution elemental mapping has become feasible by means of spatially resolved electron energy-loss spectroscopy and energy dispersive $\mathrm{x}$-ray spectroscopy in a scanning transmission electron microscope (STEM-EELS and STEM-EDX) [4-9]. At the same time, tremendous effort is being invested into the identification of the oxidation states of cations using electron energy-loss spectroscopy (EELS). The shape of the $L_{2,3}$ edge [10], the chemical shift [11,12] and the $L_{3} / L_{2}$ ratio [13] have all been used as a fingerprint for the transition metal valence. In fact, the correlation between the energy-loss near-edge structure (ELNES) and valence in different transition metal oxides was confirmed by several experiments in literature [10,14-21]. The higher the energy resolution, the more convincing this link between valence and ELNES features becomes. Combining the atomic resolution capabilities of a STEM with bonding and valence information from EELS is a highly attractive prospect. While bonding information has been obtained at atomic resolution [7,17,22], 2D oxidation state mapping at the atomic level in, e.g., metaloxide materials has remained challenging due to poor EELS signal-to-noise ratio and the need for simultaneous high spatial and energy resolution of the instrument $[10,17,23]$.
$\mathrm{Mn}_{3} \mathrm{O}_{4}$ is known to be a mixed valence compound, containing both $\mathrm{Mn}^{2+}$ and $\mathrm{Mn}^{3+}$ ions at room temperature [24]. It has a spinel structure with lattice parameters $a=5.762 \AA$ and $c=9.4696 \AA$ and space group I41/amd. Many interesting and unique properties of $\mathrm{Mn}_{3} \mathrm{O}_{4}$ warrant its applications in fields like catalysis, molecular adsorption, ion exchange, supercapacitors, magnetic applications and batteries [25-27]. The fact that it is a mixed valence compound at room temperature also makes it an ideal candidate to demonstrate valence state mapping at atomic resolution.

In this work, $150 \mathrm{~nm}$ octahedral single crystals of $\mathrm{Mn}_{3} \mathrm{O}_{4}$ were studied using atomic resolution STEMEELS [25]. The octahedral morphology of the nanocrystals allows a careful selection of the sample thickness, while the minimal presence of an amorphous surface layer enables acquisition of high-contrast images and maps [28].

The STEM-EELS experiments were performed on the FEI Titan 80-300 "cubed" microscope (Qu-Ant-EM) at EMAT [29]. Proper settings were chosen to avoid beam damage and provide the best combination of signal-tonoise ratio, spatial and energy resolution. The results were simulated with the STEMSIM software package mimicking the experimental conditions [30-33]. Detailed experimental and simulation parameters can be found in the supplementary information [28].

Figure 1 shows a high resolution high-angle annular dark-field (HAADF)-STEM image of the $\mathrm{Mn}_{3} \mathrm{O}_{4}$ sample along the [100] direction. This [100] crystal direction is chosen because the $\mathrm{Mn}^{2+}$ (in Green) and $\mathrm{Mn}^{3+}$ (in Red) cations are separated in different atomic columns (Fig. 1) [24]. The intercolumn spacing is also the largest (1.86 ̊) along this zone axis, making it the most promising for oxidation state mapping by EELS. The columns with big red circles have twice the number of ions than those 


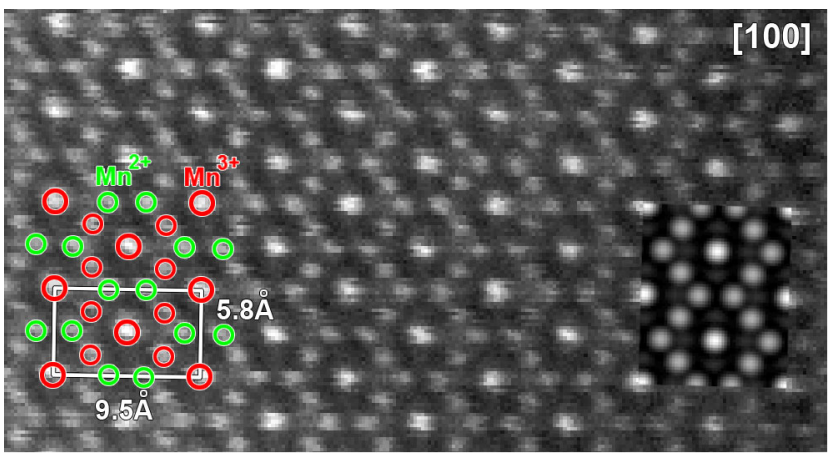

FIG. 1 (color online). High resolution HAADF-STEM image of $\mathrm{Mn}_{3} \mathrm{O}_{4}$ along the [100] direction. The cation columns are indicated by red $\left(\mathrm{Mn}^{3+}\right)$ and green $\left(\mathrm{Mn}^{2+}\right)$ circles. $A$ simulated STEM image at $16 \mathrm{~nm}$ sample thickness is inserted into the experimental image.

identified by small red and green circles, which results in a brighter contrast for these columns than for the others. However, all $\mathrm{Mn}^{3+}$ cations have the same atomic environment. Typical Fourier transforms of the acquired HAADFSTEM images display the presence of transferred frequencies down to $0.9 \AA$, meaning sub- $\AA$ information transfer is achieved [28]. A HAADF-STEM Bloch wave simulation (Fig. 1, inset) agrees well with the experimental data.

Spectroscopic data were acquired by scanning the same electron probe over the sample and simultaneously recording the EELS spectrum and the high-angle scattered electrons on a HAADF detector. This technique is commonly referred to as spectrum imaging [34]. To improve the energy resolution of the spectra, all imaging and spectroscopy was performed with a monochromator, providing an energy resolution of $0.4 \mathrm{eV}$. In the sample region where the STEM-EELS scans were performed the sample thickness was $16 \pm 2 \mathrm{~nm}$ as measured by low-loss EELS [35]. The Mn $L_{2,3}$ edge after background subtraction and averaged over the whole scan region is displayed in Fig. 2(blue). A split of the $\mathrm{Mn} L_{3}$ edge into peak $1(639.8 \mathrm{eV})$ and peak 2 $(641.5 \mathrm{eV})$ is observed, as reported for energy resolutions higher than $0.5 \mathrm{eV}[28,36,37]$. The high spatial resolution in these experiments, apparent from the simultaneously acquired HAADF-STEM image (Fig. 2, inset) enables investigation of individual atomic columns. The averaged Mn $L_{2,3}$ edges from sites of type $A\left(\mathrm{Mn}^{3+}\right)$ and $B\left(\mathrm{Mn}^{2+}\right)$ show significant differences in their ELNES. Comparing them to the average spectrum of the whole region, the $\mathrm{Mn}$ ELNES signal $B$ shows a strong enhancement of peak 1, while peak 2 is relatively weak. Vice versa for spectrum A; peak 1 is highly suppressed to a shoulder, while peak 2 is enhanced.

Comparing the Mn $L_{2,3}$ edges in $A$ and $B$ to our own references in Fig. 2 and to literature for $\mathrm{Mn}_{2} \mathrm{O}_{3}\left(\mathrm{Mn}^{3+}\right)$ and $\mathrm{MnO}\left(\mathrm{Mn}^{2+}\right)$ shows striking similarities [19,20,36,38]. Even though the chemical environment in $\mathrm{Mn}_{3} \mathrm{O}_{4}$ is

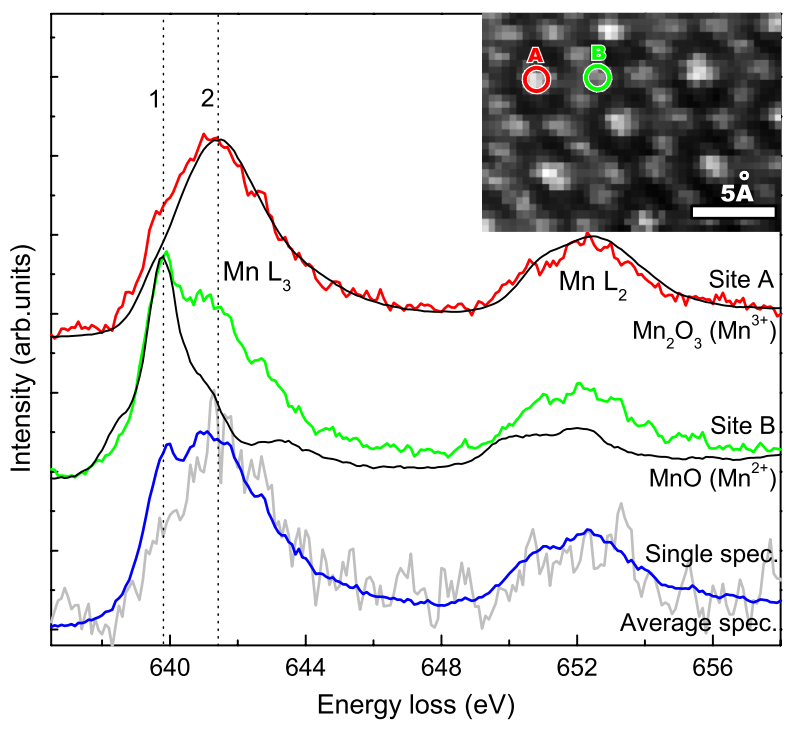

FIG. 2 (color online). Average Mn $L_{2,3}$ edge spectrum from atomic column types $A$ (red, averaged from 24 spectra) and $B$ (green, averaged from 54 spectra) together with references from $\mathrm{Mn}_{2} \mathrm{O}_{3}$ and $\mathrm{MnO}$ (black lines). Total average $\mathrm{Mn} L_{2,3}$ edge spectrum (blue) together with a single pixel spectrum (gray) from an A-type site to demonstrate the noise level. The inset shows the HAADF signal acquired simultaneously during spectrum acquisition.

different from either $\mathrm{Mn}_{2} \mathrm{O}_{3}$ or $\mathrm{MnO}$, literature has shown that the effect of valence on the ELNES signature is dominant [10,14], especially in the case of $\mathrm{Mn}$ $[17,19,20,37,38]$. Indeed the dominance of ELNES peak 1 or 2 can be attributed to $\mathrm{Mn}^{2+}$ or $\mathrm{Mn}^{3+}$ respectively as can be observed from the reference spectra. However, a discrepancy between signals $A, B$ and the references is also apparent. This can be explained in terms of intermixing of the inelastic EELS signal due to delocalization and elastic scattering [31]. This intermixing causes a fraction of $\mathrm{Mn}^{2+}$ signal to be measured at $\mathrm{Mn}^{3+}$ columns and vice versa. Moreover, the discrepancy is larger for $B$ than for $A$ owing to the fact that the total number of $\mathrm{Mn}^{3+}$ ions in $\mathrm{Mn}_{3} \mathrm{O}_{4}$ is double that of $\mathrm{Mn}^{2+}$ ions. The ELNES signatures for spectra A and B approach the reference spectra in thinner sample regions (approx. $10 \mathrm{~nm}$ ). Here, the spectra suffer less from signal intermixing but unfortunately the sample is more prone to beam damage.

Further evidence of the oxidation state of the cations in the $A$ and $B$ columns is given by the position of the $\mathrm{Mn} L_{2,3}$ edge onset. It is well known that the ionic edge of $\mathrm{Mn}$ has a higher onset at higher oxidation state $[13,17,19,20]$. This fits our experimental results well since the $\mathrm{Mn} L_{3}$ edge in $A\left(\mathrm{Mn}^{3+}\right)$ has a higher edge onset than the edge in $B\left(\mathrm{Mn}^{2+}\right)$. Besides that, the $L_{3} / L_{2}$ ratio is also higher for spectrum $B$, which is another indicator of a lower valence on $B$ sites $[13,17]$.

Using the two average spectra $A$ and $B$ as individual components in a linear combination, all the spectra in the 


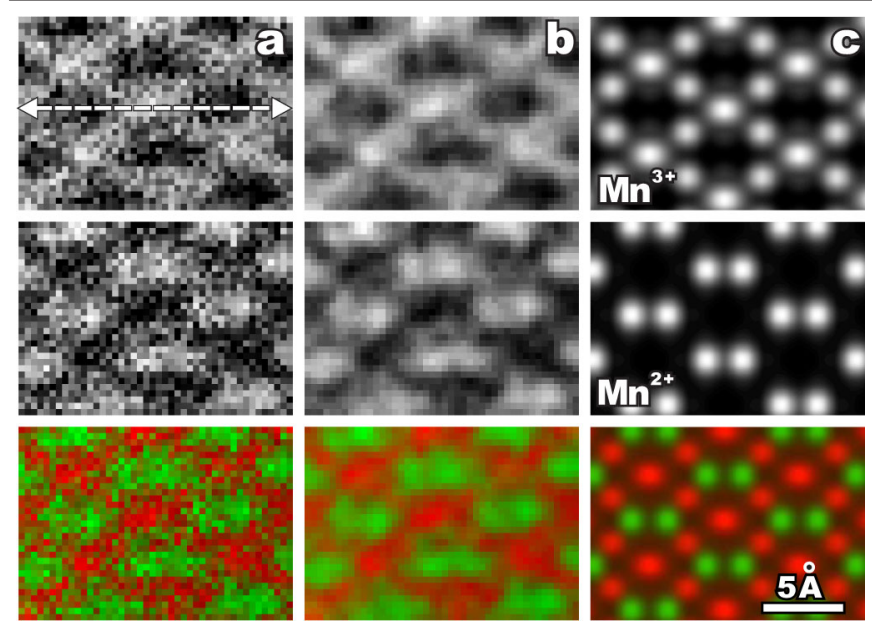

FIG. 3 (color online). Map of the Mn oxidation states in $\mathrm{Mn}_{3} \mathrm{O}_{4}$ along the [100] zone axis orientation. (a) Shows the spectral weight of component $A\left(\mathrm{Mn}^{3+}\right)$ and component $B$ $\left(\mathrm{Mn}^{2+}\right)$ obtained from spectrum fitting. A color map is displayed at the bottom $\left(\operatorname{Red}=\mathrm{Mn}^{3+}\right.$, Green $\left.=\mathrm{Mn}^{2+}\right)$. (b) Maps after low-pass filtering. (c) Simulated maps of the $\mathrm{Mn}^{3+}$ and $\mathrm{Mn}^{2+}$ signals.

SI were fitted and 2D atomic maps of the spectral weights were generated (Fig. 3). The least squares fitting to the Mn $L_{2,3}$ edges was performed using EELSMODEL [39]. We must emphasize that the analysis was performed on the raw data after background subtraction and no other data pretreatment was required as the signal-to-noise ratio is sufficient. The maps, in combination with the simultaneously acquired HAADF image (Fig. 2, inset), show that the spectral weights of $A$ and $B$ are well localized to their respective $\mathrm{Mn}^{3+}$ and $\mathrm{Mn}^{2+}$ columns [Fig. 3(a)]. For clarity, a lowpass filter was applied to improve the signal-to-noise ratio in Fig. 3(b). The $2.36 \AA$ split of neighboring $\mathrm{Mn}^{2+}$ dumbbells can be identified.

To check the validity of the local interpretation of the data, inelastic simulations at $16 \mathrm{~nm}$ thickness were performed using the STEMSIM software, making use of a double channeling approximation and a dipole model with Bethe ridge cutoff for the inelastic scattering $[30,31,40,41]$. All details of the simulation can be found in the supplementary information [28]. The simulated $\mathrm{Mn}^{3+}$ and $\mathrm{Mn}^{2+}$ maps are shown in Fig. 3(c) and match the maps for components $A$ and $B$ well in both column contrast and position. Note that the effective source size was taken into account in the simulation [42]. The simulation also shows that at this acceleration voltage and thickness the local interpretation for the $\mathrm{Mn} L_{2,3}$ edge holds and allows us to directly interpret the maps. A quantitative comparison of simulation and experiment is shown in Fig. 4 by a line profile through the experimental data.

It is important at this point to stress the difference between the weight of components $A$ and $B$ in the experiments and the simulated $\mathrm{Mn}^{3+}$ and $\mathrm{Mn}^{2+}$ signal.

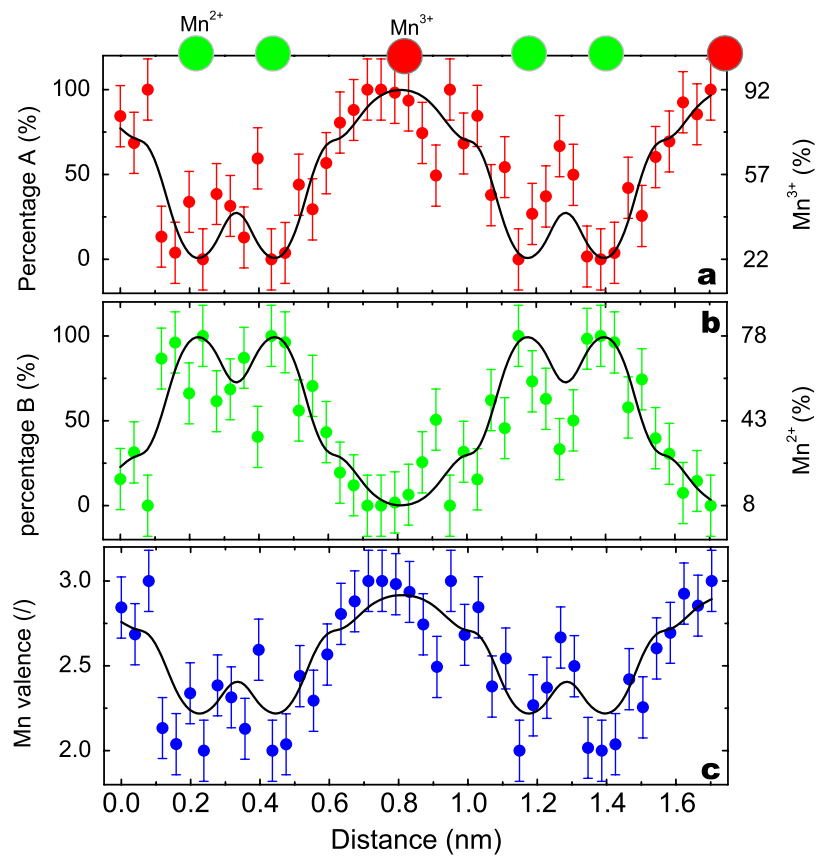

FIG. 4 (color online). (a),(b) Linear profiles of the spectral weight of component $A / B\left(\mathrm{Mn}^{3+} / \mathrm{Mn}^{2+}\right)$ taken from the single pixel line indicated by an arrow in Fig. 3. The solid lines are profiles through the simulations for a sample thickness of $16 \mathrm{~nm}$ scaled either as fraction of signal $A / B$ (left axis) or fraction of $\mathrm{Mn}^{2+} / \mathrm{Mn}^{3+}$ (right axis). The $\mathrm{Mn}^{2+}$ dumbbells (2.36 ̊) are resolved in both experiment and simulation. (c) Derived Mn valence (blue circles) obtained through allocation of spectrum $A$ to $\mathrm{Mn}^{3+}$ and spectrum $B$ to $\mathrm{Mn}^{2+}$. The solid line represents the simulated result. The effect of intermixing becomes clear through the discrepancy between the experimental and the simulated curve as explained below.

As discussed above, a certain amount of intermixing of $\mathrm{Mn}^{3+}$ and $\mathrm{Mn}^{2+}$ is present in $A$ and $B$. This means that, even though the fitted spectral weight of $A$ at $\mathrm{Mn}^{3+}$ column positions approaches $100 \%$ this does not correspond to a pure $\mathrm{Mn}^{3+}$ signal in both simulation and experiment. To take this into account, the simulated data can be rescaled to indicate either the fraction of $A$ or $B$ signal as in the experiment [Figs. 4(a) and 4(b) left axis] or to indicate the true amount of $\mathrm{Mn}^{3+} / \mathrm{Mn}^{2+}$ signal [Figs. 4(a) and 4(b) right axis]. From the latter, the simulations indicate an intermixing of approximately $8 \% \mathrm{Mn}^{2+}$ on the $A$-site and $22 \% \mathrm{Mn}^{3+}$ on the $B$ sites. This effect is also clearly observed in the experimental spectrum at site $B$ which contains a considerable fraction of $\mathrm{Mn}^{3+}$ signal (Fig. 2). Neglecting this intermixing and decomposing the experimental spectra as fractions of spectra from site $A$ and $B$ leads to an underestimate of the average valence in a complete unit cell of 2.55(1)+ which is lower than nominal value of $2.67+$ [28]. Correcting this value with the simulated intermixing leads to an average valence of $2.63(1)+$ which is only a $1 \%$ deviation from the nominal value. 
By combining both high spatial resolution $(0.9 \AA)$ and high energy resolution $(0.4 \mathrm{eV})$ in an aberration-corrected, monochromated STEM, 2D Mn oxidation state mapping has been performed on a mixed valence oxide $\mathrm{Mn}_{3} \mathrm{O}_{4}$ for the first time. The ELNES of the Mn $L_{2,3}$ edge from $\mathrm{Mn}^{2+}$ and $\mathrm{Mn}^{3+}$ cation columns is found to be similar to that for $\mathrm{MnO}$ and $\mathrm{Mn}_{2} \mathrm{O}_{3}$ references, although intermixing of the inelastic signal is found to play an important role. These experiments demonstrate the feasibility of two dimensional oxidation state mapping at atomic resolution. As a result, experiments mapping valence in, e.g., charge ordering materials can now be envisioned.

The authors acknowledge financial support from the European Union under the Framework 6 program under a contract for an Integrated Infrastructure Initiative (Reference 026019 ESTEEM). S. T. gratefully acknowledges the financial support from the Fund for Scientific Research Flanders (FWO). Thanks to Yu Li for kindly providing the sample. The Qu-Ant-EM microscope was funded by the Hercules foundation of the Flemish government. Funding from the European Research Council under the 7th Framework Program (FP7), ERC grant No. 46791-COUNTATOMS is also gratefully acknowledged.

*haiyan.tan@ua.ac.be

[1] Y. Miyamoto et al., Solid State Commun. 89, 51 (1994).

[2] D. Efremov, J. Van den Brink, and D. Khomskii, Nature Mater. 3, 853 (2004).

[3] J. van den Brink and D. I. Khomskii, J. Phys. Condens. Matter 20, 434217 (2008).

[4] E. Okunishi, H. Sawada, Y. Kondo, and M. Kersker, Microsc. Microanal. (Supp. S02), 12, 1150 (2006).

[5] M. Bosman et al., Phys. Rev. Lett. 99, 086102 (2007).

[6] K. Kimoto et al., Nature (London) 450, 702 (2007).

[7] D. A. Muller et al., Science 319, 1073 (2008).

[8] G. A. Botton, S. Lazar, and C. Dwyer, Ultramicroscopy 110, 926 (2010).

[9] A. J. Dalfonso, B. Freitag, D. Klenov, and L. J. Allen, Phys. Rev. B 81, 100101 (2010).

[10] L. F. Kourkoutis et al., Phys. Rev. Lett. 97, 256803 (2006).

[11] T. L. Daulton and B. J. Little, Ultramicroscopy 106, 561 (2006).

[12] A. M. Arevalo-Lopez and M. A. Alario-Franco, Inorg. Chem. 48, 11843 (2009).
[13] J. Rask, B. Miner, and P. Buseck, Ultramicroscopy 21, 321 (1987).

[14] N. Nakagawa, H. Hwang, and D. Muller, Nature Mater. 5, 204 (2006).

[15] L. Fitting et al., Ultramicroscopy 106, 1053 (2006).

[16] M. Varela et al., Annu. Rev. Mater. Res. 35, 539 (2005).

[17] M. Varela et al., Phys. Rev. B 79, 085117 (2009).

[18] H. Guo et al., Phys. Rev. B 79, 172402 (2009).

[19] J. Lee et al., Phys. Rev. B 80, 205112 (2009).

[20] J. H. Hwang et al., Phys. Rev. B 83, 073103 (2011).

[21] H. Tan, J. Verbeeck, A. Abakumov, and G. V. Tendeloo (to be published).

[22] K. Suenaga and M. Koshino, Nature (London) 468, 1088 (2010).

[23] L. Cave et al., Micron 37, 301 (2006).

[24] V. Baron, J. Gutzmer, H. Rundlöf, and R. Tellgren, Am. Mineral. 83, 786 (1998).

[25] Y. Li et al., Small 7, 475 (2011).

[26] M. Baldi, E. Finocchio, F. Milella, and G. Busca, Appl. Catal., B 16, 43 (1998).

[27] T. Kanasaku, K. Amezawa, and N. Yamamoto, Solid State Ionics 133, 51 (2000).

[28] See Supplemental Material at http://link.aps.org/ supplemental/10.1103/PhysRevLett.107.107602 for details of both experiment and simulation.

[29] www.emat.ua.ac.be.

[30] A. Rosenauer and M. Schowalter, in Springer Proceedings in Physics (Microscopy of Semiconducting Materials Conference, Cambridge, 2007), Vol. 120, p. 169.

[31] J. Verbeeck, P. Schattschneider, and A. Rosenauer, Ultramicroscopy 109, 350 (2009).

[32] A. Rosenauer et al., Ultramicroscopy 109, 1171 (2009).

[33] A. Rosenauer et al., Ultramicroscopy (to be published).

[34] J. Hunt and D. Williams, Ultramicroscopy 38, 47 (1991).

[35] R. F. Egerton, Electron Energy-Loss Spectroscopy in the Electron Microscope (Plenum, New York, 1996), 2nd ed.

[36] J. Paterson and O. Krivanek, Ultramicroscopy 32, 319 (1990).

[37] L. Laffont and P. Gibot, Mater. Charact. 61, 1268 (2010).

[38] L. A. J. Garvie and A. J. Craven, Phys. Chem. Miner. 21, 191 (1994).

[39] J. Verbeeck and S. V. Aert, Ultramicroscopy 101, 207 (2004).

[40] L. Allen et al., Ultramicroscopy 106, 1001 (2006).

[41] C. Dwyer, S. D. Findlay, and L. J. Allen, Phys. Rev. B 77, 184107 (2008).

[42] C. Dwyer, R. Erni, and J. Etheridge, Ultramicroscopy 110, 952 (2010). 